\section{Modification of perceptual systems}

Perceptual Modification: Adapting to Altered Sensory Environments. By R. B. Welch. (Academic: New York, San Francisco and London, 1978.) \$24.50; $£ 15.90$.

THIS excellent book provides the most comprehensive review currently available of research on adaptation of the human senses to rearrangement of the stimulus field. The author has carefully summarised the principal findings of over 500 relevant expcriments both published and unpublished, beginning with the late nineteenth century, and including important works completed only during the present year. The research methods used in each experiment are described clearly and succinctly, and arc assessed with an objective attitude that is pleasantly refreshing in a field in which evaluative reviews can often be exceedingly biased.

The greater proportion of this book is devoted to examining the diverse types of adaptation to prismaticinduced distortions of the visual field. The various mechanisms that have been proposed to account for the adaptive characteristics of the visual system are presented in detail, and their relative success in explaining the different types of adaptation to visual distortions is reviewed critically. In comparing these mechanisms, the author is careful to make an important distinction between adaptive changes occurring specifically within the visual system and adaptive changes in how vision and movement are coordinated. The failure to make that distinction has often been a source of conflicting results in previous research studies.

The outstanding sections of this book are the reviews on the necessary and sufficient conditions for adaptation to prismatic displacement, adaptation to visual transposition, adaptation to contour distortions, and differences in adaptibility among species and individuals. I was particularly impressed by the logical transition from the review of research on adaptation to inversions of the visual field to the review of research on adaptation to small angular rotations of the visual field. His treatment of these topics was notably insightful and informative; a consequence of an exceptionally clear presentation.

The superior chapter in this book, however, is that on curvature adaptation, in which the author effectively contrasts adaptive changes in visual mechanisms used for processing information about objective shape with those used for processing information about object location. The classical controversy over the role of 'active' as against 'passive' movement in leading to each type of adaptation is also treated most elegantly in that chapter.

If the book has a weakness, it would be the author's commitment to try to make sense out of each and every one of the many diverse findings that he so thoroughly summarises. As he himself points out, there seems to be an almost unlimited number of factors that can contribute to perceptual adaptation. In the final chapter, he proposes a simple theoretical model with the intention being to provide a complete account of perceptual adaptation and thereby resolve numerous controversies in the literature. Although his model does serve well as a descriptive summary of the types of conditions that lead to perceptual adaptation, it really does not provide new insights into what the fundamental underlying processes

\section{Catecholamine receptors}

Receptor Binding Studies in Adrenergic Pharmacology. By L. T. Williams and R. J. Lefkowitz. Pp. 157. (Raven: New York, \$21.45.

THE proposal by Ahlquist thirty years ago that the responses of tissues to catecholamines are mediated by either $\alpha$ - or $\beta$-adrenoceptors has formed the basis of the development of a great number of agonist and antagonist drugs for these receptor sites. It has been somewhat surprising therefore, that despite this rich selection of pharmacological probes, reliable techniques to directly identify catecholamine receptors have only been developed over the past three to four years. However, over this latter period a multitude of research papers have been published describing direct labelling techniques, and substantial advances have been made in our understanding of the characteristics and regulation of these important recognition sites.

The authors of this monograph have played major roles in the development of radiolabelled ligand binding techniques for both $\alpha$ - and $\beta$-adrenoceptors. It is therefore to be expected that much of this book describes, in considerable depth, work from their own laboratories. The authors have, however, wi itten useful introductory and linking chapters. There are succinct accounts of the pharmacology of adrenergic receptors, theory of ligandreceptor interactions and a historical perspective reviewing some of the frustrations and disappointments that were inherent in the development of these techniques. The chapter on methodo- might be. My suspicion is that there are many such processes, making an adequate model necessarily more complex than the author acknowledges.

This, however, is a minor criticism; in general, the book is an excellent summary of our current state of knowledge about the extent to which perceptual systems can be modified. It is highly recommended not only as a valuable reference for the professional researcher, but also as an informative introduction for the novice. I think the single most impressive feature of the book is the author's skill at organising an overwhclming number of isolated experiments, so as to provide the reader with a coherent account of one of the most confusing areas of visual perception.

Ronald A. Finke

Ronald A. Finke is a Spencer Foundation Predoctoral Fellow in Psychology at the Massachusetts Institute of Technology, Cambridge, Massachusetts.

logical approaches contains a few 'tricks of the trade' that may be useful for those embarking on these techniques. However, it was somewhat disappointing that discussion of atypical binding characteristics such as those associated with negative co-operativity and multiple receptor sites, are dealt with only superficially. Nevertheless, the subsequent chapters, devoted to the pharmacological characteristics of $\alpha$ - and $\beta$-adrenoceptor binding sites, are particularly detailed and emphasis is rightly placed on the importance of using radiolabelled agonists as well as antagonists.

The development of adrenergic receptor binding techniques has undoubtedly given us a new insight into the way cells may regulate their responsiveness to catecholamines. A chapter devoted entirely to this topic includes a useful account of the theoretical consequences of 'spare receptors' and the intracellular amplification of extracellular signals. Homologous and heterologous hormonal regulation of adrenergic responsiveness are discussed in some depth, though other aspects such as receptor modulation in developing tissue and genetic influences receive less attention.

Although there is no sign that the initial explosive increase in adrenergic receptor research is saturating, this book nevertheless is a timely review and the lucid style of the authors should ensure that it will become a primary and valuable source of information in adrenergic receptor pharmacology.

S. R. Nahorski

S. R. Nahorski is Lecturer in Pharmacology and Therapeutics in the School of Medicine at the University of Leicester, $U K$. 